\title{
CENTRAL MORPHISMS AND CUSPIDAL AUTOMORPHIC REPRESENTATIONS
}

\author{
JEAN-PIERRE LABESSE AND JOACHIM SCHWERMER
}

\section{INTRODUCTION}

1.1. Main theorem. Let $F$ be a global field (of arbitrary characteristic) and denote by $\mathbb{A}_{F}$ its ring of adèles. Let $G$ and $H$ be two connected reductive group defined over $F$ endowed with an $F$-morphism $f: H \rightarrow G$ such that the induced morphism $H_{d e r} \rightarrow G_{d e r}$ on the derived groups is a central isogeny. We study how automorphic representations behave, under the morphism induced between groups of adélic points, via restriction and induction. We also discuss similar statements for representations of groups over local fields.

Consider the restrictions to $f\left(H\left(\mathbb{A}_{F}\right)\right)$ of a cuspidal representation $\pi$ of $G\left(\mathbb{A}_{F}\right)$; it splits into a possibly infinite sum of irreducible representations of $H\left(\mathbb{A}_{F}\right)$ and some of them may not be automorphic. Conversely given a cuspidal representation $\sigma$ of $H\left(\mathbb{A}_{F}\right)$ it is not always possible to find it in the restriction of some cuspidal representation $\pi$ of $G\left(\mathbb{A}_{F}\right)$. Our main result is:

Theorem 1.1.1. Given any irreducible cuspidal representation $\pi$ of $G\left(\mathbb{A}_{F}\right)$ its restriction to $f\left(H\left(\mathbb{A}_{F}\right)\right)$ contains a cuspidal representation $\sigma$ of $H\left(\mathbb{A}_{F}\right)$. Conversely, assuming moreover that $f$ is an injection, any irreducible cuspidal representation $\sigma$ of $H\left(\mathbb{A}_{F}\right)$ appears in the restriction of some cuspidal representation $\pi$ of $G\left(\mathbb{A}_{F}\right)$.

Experts in the theory of automorphic forms have expected such a natural result, already known in some cases. In fact this is in agreement with Langlands functoriality conjectures which relate local or automorphic representations for a group $G$ to elements in the first cohomology set $H^{1}\left(W_{F}, \check{G}\right)$ of Weil groups with value in the dual group $\breve{G}$ and, in particular, with lifting results established in [14] for the map $H^{1}(\check{f}): H^{1}\left(W_{F}, \check{G}\right) \rightarrow H^{1}\left(W_{F}, \check{H}\right)$ when $f$ is injective.

Local results, quite elementary when the characteristic of the field is zero and already known in general to some extent (see for example 24]), are given here for the sake of completeness. In the global case, we do not know of any published reference except when $H=S L(n), G=G L(n)$. For this pair of groups, Theorem 1.1.1 (or rather its reformulation as in 5.3.4) is claimed in [17, Sect. 3] if $F$ is a number field. Unfortunately, as was pointed out by Laurent Clozel, the proof given in [17, which generalizes the argument given in [16 for $n=2$, does not apply for arbitrary $n$ since we implicitly assumed the validity of the local-global principle for the $n$-th powers in $F$, which may fail]. The argument is corrected here.

This work was partly begun during the stay of the second author in the fall term 2017 at the School of Mathematics, Institute for Advanced Study, Princeton, and then pursued at the Max-Planck-Institute for Mathematics, Bonn; he gratefully acknowledges the funding at the IAS, provided by the Charles Simonyi Endowment, as well as the support at the MPIM..

${ }^{1}$ The Grunwald-Wang Theorem [1, Chap. X, Thm. 1] computes the obstruction group to this local-global principle and, in fact, this group can be non-trivial. This only happens in very special cases: in particular $8 \mid n$ is among the necessary conditions. 
1.2. Organization of the paper. In Section 2, generalizing Clifford's theory for finite groups, we consider a pair $B \subset A$ of a locally compact groups where $B$ is a closed invariant subgroup of $A$ such that $B \backslash A$ is abelian and compact and we analyze, under a suitable finiteness condition, the interplay via restriction and induction between irreducible unitary representations $\pi$ of $A$ and $\sigma$ of $B$. Results of Section 2 are used in Section 4 to investigate extension, induction and restriction of irreducible unitary representations between pairs of groups of points over local fields of arbitrary characteristic for pairs of connected reductive groups $G$ and $H$ as above. The local results are summarizd in 4.1 .3 and 4.2 .3 Similar questions are studied in section 5 for cuspidal automorphic representations of groups of points over adèles of global fields of arbitrary characteristic and our main results are Theorems 5.2 .1 and 5.2.2. They imply in particular the above Theorem 1.1.1. We rely on structural results taken care of in Section 3 or, by a very different approach, in the appendix provided by B. Lemaire. We conclude the paper with a new multiplicity formula.

1.3. Acknowledgements. We thank Bertrand Lemaire for providing us with the appendix. We also thank Gaetan Chenevier who drew our attention to his unpublished note [6] and for pointing out a mistake in a previous version of the present note. Finally we are grateful to Guy Henniart who suggested, following his paper [11, to consider not only inclusions $H \subset G$ but also morphisms $H \rightarrow G$ inducing central isogenies between derived groups.

\section{VARIATion ON A THEME By Clifford}

In this section we establish a variant of Clifford's theory for finite groups [8] (already used implicitly in [16] or explicitly in [11 but in a slightly different context). This is elementary but, not knowing of any reference valid in our setting, it is given with some details for the convenience of the reader. In particular, we prove 2 a form of Frobenius reciprocity in 2.3.3

2.1. Notation. It is understood that unitary representations are strongly continuous and characters (i.e. one dimensional representations) are unitary. By abuse of notation we shall often denote by the same symbol a quotient and a set of representatives for its elements.

Let $A$ be a locally compact group and $B$ a closed subgroup such that $B \backslash A$ has an $A$ invariant measure. Let $\pi$ be an irreducible unitary representation of $A$ and $\sigma$ an irreducible unitary representation of $B$. Given $g \in A$ we denote by $\sigma^{g}$ the representation of $B$ defined by

$$
\sigma^{g}(x)=\sigma\left(g x g^{-1}\right) .
$$

Let $\rho$ be the induced representation

$$
\rho=\operatorname{Ind}_{B}^{A} \sigma
$$

We denote by $\langle v, w\rangle_{\sigma}$ the scalar product of two vectors $v$ and $w$ in the space $V_{\sigma}$ of the representation $\sigma$. Recall that $V_{\rho}$, the space of $\rho$, is the set of classes of measurable functions from $A$ to $V_{\sigma}$ (up to equality almost everywhere), such that $f(h g)=\sigma(h) f(g)$ and that are square integrable on $B \backslash A$.

\footnotetext{
${ }^{2}$ The reader may wonder why we give a proof of such a result since there are many references for instances where Frobenius reciprocity is known to hold. In fact, for admissible representations of reductive groups over non archimedean local fields Frobenius reciprocity is well known and could be used in certain sections below where we deal with this specific case. Nevertheless more general groups and different kinds of representations will occur and we did not find any reference for the form we need: for example Moore's result (Section 4 of [20]), which is the closest to our needs we could find, applies only to finite dimensional representations; similarly Mackey's quite general theorems (e.g. Theorem 5.1 of [18]) does not seem to be of any help since the representations we are dealing with may show up with measure zero in the spectral decomposition of the right regular representations for the groups we study.
} 
2.2. A first finiteness assumption. Assume $B \backslash A$ is of finite volume.

Lemma 2.2.1. There is an injective map

$$
\operatorname{Hom}_{B}\left(\left.\pi\right|_{B}, \sigma\right) \rightarrow \operatorname{Hom}_{A}\left(\pi, \operatorname{Ind}_{B}^{A} \sigma\right) .
$$

If $\left.\pi\right|_{B}$ and $\left.\pi^{\prime}\right|_{B}$ have a common constituent $\sigma$ then $\pi$ and $\pi^{\prime}$ both occur in $\rho=\operatorname{Ind}_{B}^{A} \sigma$.

Proof. Consider an element $\Psi \in \operatorname{Hom}_{B}\left(\left.\pi\right|_{B}, \sigma\right)$ and $w \in V_{\pi}$. The function

$$
\varphi_{w}: g \mapsto \Psi(\pi(g) w) \quad \text { for } g \in A
$$

defines a vector in $V_{\rho}$. In fact this is a continuous function which satisfies the required functional equation and whose square norm

$$
g \mapsto\left\|\varphi_{w}(g)\right\|^{2}:=<\varphi_{w}(g), \varphi_{w}(g)>_{\sigma} \leq\|\Psi\|^{2}<w, w>_{\pi}
$$

is bounded and hence integrable since $B \backslash A$ is of finite volume. The map

$$
\Phi: w \mapsto \varphi_{w}
$$

defines an element in $\operatorname{Hom}_{A}(\pi, \rho)$. The assignment $\Psi \mapsto \Phi$ is obviously injective. The second assertion follows immediately.

Assume from now on that $A$ is unimodular and $B$ is an invariant closed subgroup. The quotient group $C=B \backslash A$ is also assumed to be abelian compact and endowed with the normalized Haar measure i.e. such that $\operatorname{vol}(C)=1$. Let $X$ be the discrete group of characters of $C$. We observe that if $\pi$ occurs in $\rho=\operatorname{Ind}_{B}^{A} \sigma$ then, given $\chi \in X$, the representation $\pi \otimes \chi$ also occurs in $\rho$ with the same multiplicity.

Proposition 2.2.2. Given $\pi$ and $\pi^{\prime}$ two irreducible unitary representations of $A$ whose restrictions to $B$ have a constituent $\sigma$ in common, then there exist a character $\chi \in X$ such that

$$
\pi^{\prime} \simeq \pi \otimes \chi
$$

The representation $\rho$ is an Hilbert direct sum of representations of the form $\pi \otimes \chi$.

Proof. Since both the restrictions of $\pi$ and $\pi^{\prime}$ to $B$ have $\sigma$ as a constituent in common, Lemma 2.2.1 shows they both occur in $\rho$. Let us denote by $V_{\pi}$ the space of the representation $\pi$. Let $\Psi$ be a non-trivial intertwining operator in

$$
\operatorname{Hom}_{B}\left(\left.\pi\right|_{B}, \sigma\right)
$$

and consider for $w \in V_{\pi}$ the function

$$
\varphi_{w}: g \mapsto \Psi(\pi(g) w)
$$

The closed subspace generated by the functions $\varphi_{w} \chi$, where $w$ varies in $V_{\pi}$ and $\chi$ varies in $X$, is the space of a subrepresentation $\rho^{\prime}$ of $\rho$, generated by a set of subrepresentations isomorphic to $\pi \otimes \chi$. Let $f$ be a function from $A$ to $V_{\sigma}$ that belongs to the orthogonal $\rho^{\prime \prime}$ of $\rho^{\prime}$. We have to show that $f=0$. Let us denote by $\langle\varphi, f\rangle_{\rho}$ the scalar product of two functions $\varphi$ and $f$ in the space of $\rho$. By hypothesis

$$
<\varphi_{w} \chi, f>_{\rho}=\int_{C} \chi(g)<\varphi_{w}(g), f(g)>_{\sigma} d \dot{g}=0
$$

for all $w \in V_{\pi}$ and all $\chi \in X$. This implies that $\left\langle\varphi_{w}(g), f(g)\right\rangle_{\sigma}=0$ for almost all $\dot{g} \in C$ and all $w \in V_{\pi}$. Now $w \mapsto \varphi_{w}(g)$ is an intertwining operator $\Psi^{g}$ between $\left.\pi\right|_{B}$ and $\sigma^{g}$, a representation of $B$ in $V_{\sigma}$ which is irreducible; the image of $\Psi^{g}$ equals $V_{\sigma}$ and necessarily $f(g)=0$ for almost all $g$. 
2.3. A second finiteness assumption. We denote by $A(\sigma)$ the subgroup of $A$ (containing $B$ ) of $g \in A$ such that $\sigma^{g} \simeq \sigma$ and by $X(\pi)$ the subgroup of $\chi \in X$ such that $\pi \otimes \chi \simeq \pi$. We shall now moreover assume that $X(\pi)$ is finite.

Proposition 2.3.1. Let $\pi$ be an irreducible unitary representation of $A$ such that $X(\pi)$ is finite. Its restriction $\left.\pi\right|_{B}$ is a finite direct sum of irreducible unitary representations of $B$. Let $\sigma$ be an irreducible constituent of $\left.\pi\right|_{B}$. The vector space

$$
V=\operatorname{Hom}_{B}\left(\left.\pi\right|_{B}, \sigma\right)
$$

is of finite dimension, say $m$. All other constituents are conjugates under $A$ of $\sigma$ and

$$
\left.\pi\right|_{B} \simeq \bigoplus_{\dot{g} \in A / A(\sigma)} V \otimes \sigma^{g}
$$

where $B$ acts trivially on $V$. The algebra $\mathcal{I}(\pi)$, of intertwining operators for $\pi$ restricted to $B$, has a basis indexed by $X(\pi)$ and

$$
\operatorname{dim}(\mathcal{I}(\pi))=\operatorname{card}(X(\pi))=m^{2} \times \operatorname{card}(A / A(\sigma)) .
$$

Proof. For $\chi \in X(\pi)$ choose a non-trivial intertwining operator $U_{\chi}$ between $\pi$ and $\pi \otimes \chi$. According to Schur's lemma, the operator $U_{\chi}$ is well defined up to a scalar. Consider $I \in \mathcal{I}(\pi)$ and $\chi \in X$, then the operator

$$
I_{\chi}=\int_{\dot{g} \in C} \overline{\chi(g)} \cdot \pi(g)^{-1} I \pi(g) d \dot{g}
$$

is a scalar multiple of $U_{\chi}$ for $\chi \in X(\pi)$ and is zero if $\chi \notin X(\pi)$. Fourier inversion shows that

$$
I=\sum_{\chi \in X(\pi)} I_{\chi}=\sum_{\chi \in X(\pi)} c_{\chi} U_{\chi}
$$

with $c_{\chi} \in \mathbb{C}$. This implies $\operatorname{dim}(\mathcal{I}(\pi))=\operatorname{card}(X(\pi))$. By assumption $\sigma$ is an irreducible constituent of $\left.\pi\right|_{B}$. The closed subspace generated by the isotypic components of $\sigma$ and its $A$-conjugates is an $A$-invariant subspace of $V_{\pi}$, equal to $V_{\pi}$ since $\pi$ is irreducible. Hence $\left.\pi\right|_{B}$ is isomorphic to a finite sum of irreducible representations of $B$ that are $A$-conjugates of $\sigma$.

The group $X(\pi)$ is of course finite when $C$ is finite but there are many other instances of it, in particular when dealing with admissible representations (see 4.1.1 and 5.1.1 below).

One should note that the algebra $\mathcal{I}(\pi)$ may not be isomorphic to the group algebra $\mathbb{C}[X(\pi)]$. This is the case when $m \geq 2$. An example occurs in the study of inner forms of $S L(2)$ (cf. [16]) where one may have $A(\sigma)=A$ while $X(\pi)$ is an abelian group of order 4 but $m=2$ and $\mathcal{I}(\pi)=M(2, \mathbb{C})$ the algebra of $2 \times 2$ matrices. Further examples are given in [12].

Consider the subgroup $B(\pi)$ of $g \in A$ such that $\chi(g)=1$ for all $\chi \in X(\pi)$. If $X(\pi)$ is finite, $B(\pi)$ is of index $\operatorname{card}(X(\pi))$ in $A$ and we have the following inclusions

$$
B \subset B(\pi) \subset A(\sigma) \subset A .
$$

Corollary 2.3.2. If $X(\pi)$ is finite the representation $\sigma$ of $B$ can be extended to a representation $\tilde{\sigma}$ of $B(\pi)$ in the same space.

Proof. Proposition 2.3.1 applied to the pairs $(A, B)$ and $(A, B(\pi))$ tells us that the dimension of the intertwining algebra for $\left.\pi\right|_{B(\pi)}$ and $\left.\pi\right|_{B}$ are both equal to $\operatorname{card}(X(\pi))$ and hence the irreducible constituents of $\left.\pi\right|_{B(\pi)}$ remain irreducible when restricted to $B$. 
Proposition 2.3.3. Let $B \subset A$ be a pair of a locally compact groups where $B$ is a closed invariant subgroup of $A$ such that $B \backslash A$ is a compact abelian group. Let $\pi$ and $\sigma$ be irreducible unitary representations of $A$ and $B$ respectively. Assume that the group $X(\pi)$, of characters of $B \backslash A$ such that $\pi \otimes \chi \simeq \pi$, is finite. Then Frobenius reciprocity holds: the natural map

$$
\text { Frob : } \operatorname{Hom}_{B}\left(\left.\pi\right|_{B}, \sigma\right) \rightarrow \operatorname{Hom}_{A}\left(\pi, \operatorname{Ind}_{B}^{A} \sigma\right) \text {. }
$$

is an isomorphism.

Proof. We have seen that $\sigma$ can be extended to a representation $\tilde{\sigma}$ of $B(\pi)$. Since $A / B(\pi)$ is finite all functions in the space of $\operatorname{Ind}_{B(\pi)}^{A} \tilde{\sigma}$ are continuous and evaluation at the origin yields the Frobenius reciprocity, i.e. the following map is a bijection:

$$
\operatorname{Hom}_{B(\pi)}\left(\left.\pi\right|_{B(\pi)}, \tilde{\sigma}\right) \rightarrow \operatorname{Hom}_{A}\left(\pi, \operatorname{Ind}_{B(\pi)}^{A} \tilde{\sigma}\right) .
$$

On the other hand, there is an isomorphism

$$
\operatorname{Hom}_{B}\left(\left.\pi\right|_{B}, \sigma\right) \rightarrow \operatorname{Hom}_{B(\pi)}\left(\left.\pi\right|_{B(\pi)}, \tilde{\sigma}\right) .
$$

Now $\tilde{\sigma}$ injects in $\operatorname{Ind}_{B}^{B(\pi)} \sigma$ : in fact the map

$$
w \mapsto f_{w} \quad \text { with } \quad f_{w}(x)=\tilde{\sigma}(x) w
$$

is an intertwining operator since

$$
\rho(y) f_{w}(x)=f_{w}(x y)=\tilde{\sigma}(x y) w=f_{\tilde{\sigma}(y) w}(x),
$$

and it follows form 2.2.2 that $\operatorname{Ind}_{B}^{B(\pi)} \sigma$ is the Hilbert direct sum of the $\tilde{\sigma} \otimes \nu$ where $\nu$ runs over characters of $B(\pi) / B$ while $\operatorname{Ind}_{B(\pi)}^{A} \tilde{\sigma}$ is a multiple of $\pi$. This implies that

$$
\operatorname{Hom}_{A}\left(\pi, \operatorname{Ind}_{B(\pi)}^{A}(\tilde{\sigma} \otimes \nu)\right)=\operatorname{Hom}_{A}\left(\pi,\left(\operatorname{Ind}_{B(\pi)}^{A} \tilde{\sigma}\right) \otimes \chi\right)=0
$$

unless $\chi$, which is any extension of $\nu$ to $A$, belongs to $X(\pi)$. Now induction by stages shows that

$$
\operatorname{Ind}_{B}^{A} \sigma=\widehat{\bigoplus}_{\nu} \operatorname{Ind}_{B(\pi)}^{A}(\tilde{\sigma} \otimes \nu)
$$

where $\nu$ runs over characters of $B(\pi) / B$ and (c) implies

$$
\operatorname{Hom}_{A}(\pi, \rho)=\operatorname{Hom}_{A}\left(\pi, \operatorname{Ind}_{B}^{A} \sigma\right)=\operatorname{Hom}_{A}\left(\pi, \operatorname{Ind}_{B(\pi)}^{A} \tilde{\sigma}\right)
$$

In view of (a), (b) and (d) the proof is complete.

\section{The GRoups IN QUESTION}

Let $k$ be a field. Let $H$ and $G$ be two connected algebraic groups over $k$ with a morphism $f: H \rightarrow G$. Let $Z^{G}$ denote the center of $G$ and $Z^{H}$ the center of $H$. Let $Z$ be the connected component of $Z^{G}$; this is a torus.

3.1. Some crossed modules. We shall assume that the natural morphism $Z \times H \rightarrow G$ is a central map which means that it is surjective and its kernel an abelian group scheme in the center (see Appendix A). This is equivalent to ask that the morphism induced between the derived subgroups

$$
f_{\text {der }}: H_{\text {der }} \rightarrow G_{\text {der }}
$$

is a central isogeny. This is also equivalent to asking that the induced map

$$
f_{a d}: H_{a d} \rightarrow G_{a d}
$$

between the adjoint groups is an isomorphism. The last isomorphism shows that $G$ acts on $H$ by conjugacy and this implies that the complex $[H \rightarrow G]$ is a crossed-module. We refer the reader to [15, Chap. 1] or [19, Appendix B] for this concept. The particular case where 
$H=G_{s c}$ is the simply connected cover of the derived group has been extensively studied in [15.

Lemma 3.1.1. Let $T^{G}$ be a maximal torus in $G$ and let $T^{H}$ be its inverse image in $H$. The map between complexes

$$
\left[T^{H} \rightarrow T^{G}\right] \rightarrow[H \rightarrow G]
$$

induces a quasi-isomorphism between complexes of points over the separable closure.

Proof. Let $k^{\text {sep }}$ denote the separable closure of $k$. We want to prove that

$$
\left[T^{H}\left(k^{s e p}\right) \rightarrow T^{G}\left(k^{s e p}\right)\right] \rightarrow\left[H\left(k^{s e p}\right) \rightarrow G\left(k^{s e p}\right)\right]
$$

is a quasi-isomorphism. In particular we need to compute the kernel and cokernel of the map $f^{s e p}$. But then we are dealing with split groups and split tori. Since the unipotent subgroups and Weyl groups are isomorphic [5, Théorème (2.20), page 260] and using Bruhat decomposition, we are left with the kernel and cokernel of the induced map between the tori.

3.2. Crossed modules over local fields. In this subsection $F$ is a local field; by this we mean archimedean or non archimedean local field as well. As a convention for the Galois cohomology with values in complexes $[B \rightarrow A]$ we take $A$ in degree 0 . This is the convention used in [15].

We denote by $H^{+}$the subgroup of $G(F)$ generated by $f(H(F))$ and $Z^{G}(F)$. The reader is warned that although $H^{+}$is a Lie group when $F$ is archimedean and a totally disconnected group when $F$ is non archimedean, it is not in general the group of points of an algebraic reductive group over $F$.

Proposition 3.2.1. The group $H^{+}$is an invariant subgroup in $G(F)$. The quotient $G(F) / H^{+}$ is abelian and compact; it is even finite for local fields of characteristic zero.

Proof. Replacing if necessary $H$ by $H \times Z$ which is again reductive and connected, we may assume that the map $f$ is surjective. Then it is enough to prove that, in such a case, $f(H(F)) \backslash G(F)$ is abelian, compact and even finite for local fields of characteristic zero. Since quasi-isomorphisms between complexes of points on the separable closure compatible with Galois action induce isomorphisms in Galois cohomology [15, Proposition 1.2.2], Lemma 3.1.1 implies that the map

$$
\mathbf{H}^{0}\left(F,\left[T^{H} \rightarrow T^{G}\right]\right) \rightarrow \mathbf{H}^{0}(F,[H \rightarrow G])
$$

is an isomorphism and hence $\mathbf{H}^{0}(F,[H \rightarrow G])$ is abelian. One has an exact sequence

$$
1 \rightarrow f(H) \backslash G \rightarrow \mathbf{H}^{0}(F,[H \rightarrow G]) \rightarrow \mathbf{H}^{1}(F, H) .
$$

In particular $f(H) \backslash G$ is an abelian subgroup of finite index in $\mathbf{H}^{0}(F,[H \rightarrow G])$. There is an exact sequence

$$
1 \rightarrow f\left(T^{H}\right) \backslash T^{G} \rightarrow \mathbf{H}^{0}\left(F,\left[T^{H} \rightarrow T^{G}\right]\right) \rightarrow \mathbf{H}^{1}\left(F, T^{H}\right) .
$$

Since $\mathbf{H}^{1}\left(F, T^{H}\right)$ is finite it remain to observe that $f\left(T^{H}\right) \backslash T^{G}$ is compact when $f$ is surjective (see for example Lemma A.1.1 in Appendix A). It is finite for local fields of characteristic zero

For an alternative argument independent of Galois hypercohomology see A.2.1 in Appen$\operatorname{dix}$ A.

Remark 3.2.2. In the case of a central isogeny $H \rightarrow G$ for groups over a non-archimedean local field this result was stated (without proof) and used in [22]. 
3.3. Crossed modules over global fields. In this subsection $F$ is a global field. We shall use the notation of [13] and [15] for adelic cohomology. The reader should be aware that the degree conventions for hypercohomology of complexes are not the same in these references: namely $\mathbf{H}^{0}(\star, B \rightarrow A)$ in [15] is $\mathbf{H}^{1}(\star, B \rightarrow A)$ in [13]. We shall use the convention of [15].

Lemma 3.3.1. Assume the morphism $f: H \rightarrow G$ is surjective. Then $\mathbf{H}^{0}\left(\mathbb{A}_{F} / F,[H \rightarrow G]\right)$ is compact.

Proof. The quasi-isomorphism $\left[T^{H} \rightarrow T^{G}\right] \rightarrow[H \rightarrow G]$ implies isomorphisms in cohomology. Hence it is equivalent to prove that

$$
\mathbf{H}^{0}\left(\mathbb{A}_{F} / F,\left[T^{H} \rightarrow T^{G}\right]\right)
$$

is compact. However, this is one of the statements in Lemma C.2.D, page 153, in [13] (up to the shift in degree explained above). Although this reference is written for number fields the proof extends verbatim to the case of arbitrary global fields. Namely, one has an exact sequence

$$
1 \rightarrow D \rightarrow \mathbf{H}^{0}\left(\mathbb{A}_{F} / F,\left[T^{H} \rightarrow T^{G}\right]\right) \rightarrow \mathbf{H}^{1}\left(\mathbb{A}_{F} / F, T^{H}\right)
$$

where

$$
D=\operatorname{Coker}\left[\mathbf{H}^{0}\left(\mathbb{A}_{F} / F, T^{H}\right) \rightarrow \mathbf{H}^{0}\left(\mathbb{A}_{F} / F, T^{G}\right)\right]
$$

is compact if $f$ is surjective while $\mathbf{H}^{1}\left(\mathbb{A}_{F} / F, T^{H}\right)$ is finite.

Lemma 3.3.2. Assume $f$ is surjective. Then $G(F) f\left(H\left(\mathbb{A}_{F}\right)\right) \backslash G\left(\mathbb{A}_{F}\right)$ is compact.

Proof. Let us denote by $K$ the complex $[H \rightarrow G]$. The following diagram

$$
\begin{aligned}
& H(F) \quad \rightarrow \quad H\left(\mathbb{A}_{F}\right) \quad \rightarrow \quad \mathbf{H}^{0}\left(\mathbb{A}_{F} / F, H\right) \\
& \downarrow \quad \downarrow \quad \downarrow \\
& G(F) \quad \rightarrow \quad G\left(\mathbb{A}_{F}\right) \quad \rightarrow \quad \mathbf{H}^{0}\left(\mathbb{A}_{F} / F, G\right) \\
& \begin{array}{c}
\downarrow \\
\mathbf{H}^{0}(F, K) \rightarrow \mathbf{H}^{0}\left(\mathbb{A}_{F}, K\right)
\end{array} \rightarrow \mathbf{H}^{0}\left(\begin{array}{l}
\downarrow \\
\left.\mathbb{A}_{F} / F, K\right)
\end{array} \rightarrow \operatorname{Ker}^{1}(F, K)\right. \\
& \operatorname{Ker}^{1}(F, H) \rightarrow \mathbf{H}^{0}(F, H) \rightarrow \mathbf{H}^{0}\left(\mathbb{A}_{F}^{\downarrow}, H\right)
\end{aligned}
$$

is commutative with exact lines and columns. Now 3.3.1 and the finiteness of

$$
\operatorname{Ker}^{1}(F, K) \simeq \operatorname{Ker}^{1}\left(F,\left[T^{H} \rightarrow T^{G}\right]\right)
$$

(cf. [13) imply that

$$
\operatorname{Coker}\left[\mathbf{H}^{0}(F,[H \rightarrow G]) \rightarrow \mathbf{H}^{0}\left(\mathbb{A}_{F},[H \rightarrow G]\right)\right]
$$

is also compact. Thanks to the finiteness of $\operatorname{Ker}^{1}(F, H)$ (cf. [15, Prop. 1.7.3] for number fields, which rephrases results of Kottwitz and [7, Thm 1.3.3] for function fields. The latter one relies on [10]) the image of $G\left(\mathbb{A}_{F}\right)$ in this cokernel is up to a finite subgroup isomorphic to the quotient

and hence this quotient is also compact.

$$
G(F) f\left(H\left(\mathbb{A}_{F}\right)\right) \backslash G\left(\mathbb{A}_{F}\right)
$$

We now return to the general case where $f: H \rightarrow G$ need not be surjective.

Proposition 3.3.3. Let $H^{+}:=Z^{G}\left(\mathbb{A}_{F}\right) G(F) f\left(H\left(\mathbb{A}_{F}\right)\right)$. The quotient $H^{+} \backslash G\left(\mathbb{A}_{F}\right)$ is an abelian compact group.

Proof. Replacing if necessary $H$ by $H \times Z$ this is a consequence of Lemma 3.3 .2

For an alternative argument independent of adèlic hypercohomology see $\mathrm{A.2.2}$ in Appen$\operatorname{dix} \mathrm{A}$. 


\section{A first application of Clifford's theory: THE LOCAL CASE}

In this section $F$ is a local field. Some aspects of what follows have been observed by various authors (see in particular [9], [11, [12, [16], [17, [22] and [24]).

4.1. The basic results. Consider $G$ and $H$ with a map $f: H \rightarrow G$ over $F$ inducing a central isogeny of their derived groups and consider $H^{+}$the subgroup of $G(F)$ generated by $f(H(F))$ and $Z^{G}(F)$. We denote by $N$ the kernel of the map $f: H \rightarrow G$.

Lemma 4.1.1. The quotient $H^{+} \backslash G(F)$ is abelian compact. If $\pi$ is an irreducible unitary representation of $G(F)$ then the group $X(\pi)$ of characters $\chi$ of $H^{+} \backslash G(F)$ such that $\pi \otimes \chi \cong \pi$ is finite.

Proof. We apply the results of section 2 to $A=G(F)$ and $B=H^{+}$. The assertions are obvious when $C=H^{+} \backslash G$ is finite which is the case for local fields of zero characteristic according to Proposition 3.2.1 For non archimedean fields of arbitrary characteristic we appeal again to Proposition 3.2.1 or A.2.2 for the first statement. The finiteness of $X(\pi)$ is known for admissible irreducible representations ([11], [22]). To conclude we recall that the subspace of smooth vectors in an irreducible unitary representation of the group of points of a connected reductive group group over a non archimedean local field is admissible ([2]).

Let $F$ be a non archimedean local field, and assume $G$ is a quasi-split connected reductive group, split over an unramified extension. Choose an hyper-special maximal compact subgroup $K \subset G(F)$. We say that a representation $\pi$ of $G(F)$ is unramified if the operator $\pi(K)$ fixes a non zero vector.

Lemma 4.1.2. If $\pi$ is unramified, all elements in $X(\pi)$ are also unramified.

Proof. Choose an Iwahori subgroup $I \subset K$; then there is a unique Borel subgroup $P_{0} \subset G$ with Levi decomposition $P_{0}=T \ltimes U$ such that

$$
I=(T(F) \cap I)(U(F) \cap I)(\bar{U}(F) \cap I)
$$

where $\bar{U}$ is the opposite unipotent subgroup. An unramified representation $\pi$ is the spherical subquotient of a principal series representation obtained by parabolic induction of a character $\lambda$ of $T(F)$ which is trivial on $T(F) \cap I$. A character $\chi \in X(\pi)$ defines by restriction a character $\tilde{\chi}$ of $T(F)$. The representation $\pi \otimes \chi$ is a subquotient of the principal series representation obtained by parabolic induction of $\lambda \tilde{\chi}$. But since $\pi \simeq \pi \otimes \chi$ one has

$$
\lambda \tilde{\chi}=s(\lambda) \quad \text { for some } s \text { in the Weyl group . }
$$

This shows that $\tilde{\chi}=s(\lambda) \lambda^{-1}$ is trivial on $T(F) \cap I$. Then $\chi$ must be trivial on the subgroup generated by $f(H(F))$ and $T(F) \cap I$. Now $f(H(F)) \supset U(F) \supset U(F) \cap I$ and similarly for $\bar{U}$. Hence $\chi$ is trivial on $I$. Denote by $K^{\prime}$ the hyper-special subgroup in $H(F)$ such that $f\left(K^{\prime}\right) \subset K$. Any $s^{\prime} \in W^{\prime}$ has a representative $w_{s^{\prime}} \in H(F) \cap K^{\prime}$. The Weyl group $W^{\prime}$ of $H(F)$ maps bijectively via $f$ onto the Weyl group $W$ of $G(F)$ and hence any $s \in W$ has a representative

$$
w_{s}=f\left(w_{s^{\prime}}\right) \in f(H(F)) \cap K .
$$

Since the $w_{s}$ and $I$ generate $K$ the character $\chi$ is trivial on $K$.

Proposition 4.1.3. Given an irreducible unitary representation $\pi$ of $G(F)$ its restriction to $H(F)$ is a direct sum of finitely many irreducible unitary representations that are $G(F)$ conjugate. Conversely, any irreducible unitary representation of $H(F)$ trivial on $N$ occurs in the restriction of some $\pi$ and all such irreducible representations are of the form $\pi \otimes \chi$ with $\chi \in X$ the group of characters of $G(F) / H^{+}$. 
Proof. We first restrict $\pi$ to $H^{+}$. In view of Lemma 4.1.1 we may use Proposition 2.3.1 with $A=G(F)$ and $B=H^{+}$. Hence this restriction is a direct sum of finitely many irreducible unitary representations of $H^{+}$that are conjugate under $G(F)$. Then restriction from $\mathrm{H}^{+}$ to $N \backslash H(F)$ preserves irreducibility.

Conversely, consider a representation $\sigma$ of $H(F)$ and $\omega$ a character of $Z^{G}(F)$ such that its restriction to $Z^{H}(F)$ is the character with which $Z^{H}(F)$ acts via $\sigma$. One can extend $\sigma$ to a representation $\sigma^{+}$of $H^{+}$and then induce this representation from $H^{+}$to $G(F)$. According to Proposition 2.2.2 this is a sum of representations of the form $\pi \otimes \chi$ with $\pi$ irreducible and $\chi \in X$ the group of characters of $H^{+} \backslash G(F)$. The restriction of $\pi$ to $N \backslash H(F)$ contains $\sigma$ according to Proposition 2.3.3.

We observe that if $G$ and $H$ are quasisplit, and if $\pi$ is generic (i.e. has a Whittaker model for some character of the unipotent radical of a chosen Borel subgroup) the restriction $\left.\pi\right|_{f(H(F))}$ is multiplicity free (i.e. $m=1$ in the notation of Proposition 2.3.1) as follows from Proposition 2.3 .3 using the uniqueness of Whittaker models and the compatibility of Whittaker models with induction.

\subsection{Two equivalence relations.}

Definition 4.2.1. We say that two irreducible unitary representations $\sigma$ and $\sigma^{\prime}$ of $H(F)$ are in the same "G(F)-packet" if there exists an element $g \in G(F)$ such that $\sigma^{g} \cong \sigma^{\prime}$. We denote by $\mathcal{A}_{G}(H)$ the set of $G(F)$-packets of irreducible unitary representations of $H(F) / N$.

We observe that $G(F)$-packets coincide with $L$-packets when $H=S L(n)$ and $G=G L(n)$ and for compatible inner forms as well. In general $L$-packets should be unions of $G(F)$ packets since adjoint conjugacy is a special case of stable conjugacy.

Definition 4.2.2. We define two irreducible unitary representations $\pi$ and $\pi^{\prime}$ of $G(F)$ to be $\mathcal{E}_{H}$-equivalent if there exists a character $\mu$ of $G(F) / H^{+}$such that $\pi \otimes \mu \cong \pi^{\prime}$. We denote by $\mathcal{E}_{H}(G)$ the corresponding set of equivalence classes.

Now, all elements in the $\mathcal{E}_{H}$-class of some $\pi$ have equivalent restrictions to $f(H(F))$ and all components of the restriction belong to the same $G$-packet. Let $R$ be the map which assigns to an $\mathcal{E}_{H}$-equivalence class represented by $\pi$ the $G(F)$-packet of components $\sigma^{g}$ of the restriction of $\pi$ to $H(F)$. The above Propositions and remarks can be summarized as

Proposition 4.2.3. The map $R: \mathcal{E}_{H}(G) \rightarrow \mathcal{A}_{G}(H)$ is a bijection.

\section{Second application: the Case of Cuspidal Representations.}

Now $F$ is a global field and we examine how cuspidal automorphic representation behave under restriction and induction. By cuspidal representation we understand an irreducible unitary automophic representation ocuring in the cuspidal spectrum. For a definition of these objects over fields of arbitrary characteristics we refer the reader to 21]. We consider two connected reductive groups with a map $f: H \rightarrow G$ over some global field $F$ inducing a central isogeny of their derived groups.

5.1. The key construction. We have introduced in subsection 3.3 the subgroup

$$
H^{+}:=Z^{G}\left(\mathbb{A}_{F}\right) G(F) f\left(H\left(\mathbb{A}_{F}\right)\right)
$$

in $G\left(\mathbb{A}_{F}\right)$. According to Propositions 3.3.3 or A.2.2 the quotient $H^{+} \backslash G\left(\mathbb{A}_{F}\right)$ is abelian and compact. 
Lemma 5.1.1. Let $\pi$ be an automorphic representation of $G\left(\mathbb{A}_{F}\right)$. Then the group $X(\pi)$ of characters of $H^{+} \backslash G\left(\mathbb{A}_{F}\right)$ such that $\pi \otimes \chi \simeq \pi$ is finite.

Proof. We observe that $H^{+}$contains the product over all places $v$ of groups $H_{v}^{+}$generated by $f\left(H_{v}\right)$ and $Z_{v}^{G}$. Let $\pi$ be an automorphic representation of $G\left(\mathbb{A}_{F}\right)$. Thanks to Lemma 4.1 .1 and 4.1 .2 we know there is a compact open subgroup $K_{f}$ of the finite adèles on which any $\chi \in X(\pi)$ is trivial. Recall that $H_{\infty}^{+}$is of finite index in $G_{\infty}$ when $F$ is a number field. In all cases $K_{f} \cdot H^{+}$is an open subgroup of finite index in $G\left(\mathbb{A}_{F}\right)$ on which any $\chi$ such that $\pi \otimes \chi \simeq \pi$ is necessarily trivial, hence $X(\pi)$ is finite.

Denote by $\mathbf{N}$ the kernel of the map $f_{\mathbb{A}_{F}}: H\left(\mathbb{A}_{F}\right) \rightarrow G\left(\mathbb{A}_{F}\right)$. This is a subgroup in the center of $H\left(\mathbb{A}_{F}\right)$ and we may identify $\mathbf{N} \backslash H\left(\mathbb{A}_{F}\right)$ with $f\left(H\left(\mathbb{A}_{F}\right)\right)$. Let $Z^{+}:=Z^{G}\left(\mathbb{A}_{F}\right) G(F)$. Observe that

$$
Z^{+} / G(F)=Z^{G}\left(\mathbb{A}_{F}\right) G(F) / G(F)=Z^{G}\left(\mathbb{A}_{F}\right) / Z^{G}(F) .
$$

Let

$$
Z_{1}^{+}: Z^{+} \cap f\left(H\left(\mathbb{A}_{F}\right)\right) \quad \text { and } \quad Z_{1}=f^{-1}\left(Z_{1}^{+}\right) .
$$

$Z_{1}$ is a closed subgroup in $H\left(\mathbb{A}_{F}\right)$ that contains and normalizes $H(F)$. Let

$$
\Gamma^{+}=G(F) \cap Z_{1}^{+}=G(F) \cap f\left(H\left(\mathbb{A}_{F}\right)\right) \quad \text { and } \quad \Gamma=f^{-1}\left(\Gamma^{+}\right) .
$$

The subgroup $\Gamma$ in $H\left(\mathbb{A}_{F}\right)$ contains $\mathbf{N} . H(F)$ and $Z_{1}^{+} / \Gamma^{+} \simeq Z_{1} / \Gamma$. Thus, a unitary character

$$
\omega: Z^{G}\left(\mathbb{A}_{F}\right) / Z^{G}(F) \rightarrow \mathbb{C}^{\times}
$$

defines a character of $Z^{+}$, again denoted $\omega$, and we obtain by restriction a character $\omega_{1}^{+}$on $Z_{1}^{+}$trivial on $\Gamma^{+}$. Observe that conversely any character on $Z_{1}^{+} / \Gamma^{+}$extends to a character of $Z^{G}\left(\mathbb{A}_{F}\right) / Z^{G}(F)$. Denote by $\omega_{1}$ the character of $Z_{1} / \Gamma$ defined by $\omega_{1}^{+}$.

Remark 5.1.2. Observe that if $f$ is injective, i.e. if $H$ is a subgroup of $G$, then

$$
\Gamma=G(F) \cap H\left(\mathbb{A}_{F}\right)=H(F) .
$$

But when $f$ is not injective it may happen that $\mathbf{N} . H(F)$ is a strict subgroup of $\Gamma$. This is, for example, the case if $G=\mathbb{G}_{m}, H=\mathbb{G}_{m}$ and $f: x \mapsto x^{n}$ when $(F, n)$ is a counter example to the local-global principle for $n$-th powers (see [1, Chap. X, Thm. 1]).

Since the group $Z_{1}$ normalizes $H(F)$, it acts via left translations on $H(F) \backslash H\left(\mathbb{A}_{F}\right)$, hence on the space

$$
L^{2}\left(H(F) \backslash H\left(\mathbb{A}_{F}\right), \omega_{0}\right)
$$

of functions that are square-integrable modulo the center on $H(F) \backslash H\left(\mathbb{A}_{F}\right)$ and that transforms according to $\omega_{0}$ some automorphic character of the center of $H\left(\mathbb{A}_{F}\right)$. The latter space is endowed with the right regular representation $\rho_{\omega_{0}}$ of $H\left(\mathbb{A}_{F}\right)$.

The space of left $\Gamma$ invariant functions that are square-integrable modulo the center on $H(F) \backslash H\left(\mathbb{A}_{F}\right)$ can be decomposed according to the characters of $\Gamma \backslash Z_{1}$ and this decomposition is compatible with the spectral decomposition of the right regular representation. Observe that the action of $Z_{1}$ preserves cuspidality. Now, given $\omega$ and $\omega_{1}$ as above consider a function $\varphi$ on $H\left(\mathbb{A}_{F}\right)$ which satisfies the condition

$$
\varphi(c h)=\omega_{1}(c) \varphi(h) \text { for all } c \in Z_{1}, h \in H\left(\mathbb{A}_{F}\right) .
$$

There exists a unique function $\varphi^{+}$on $H^{+}$such that

$$
\varphi^{+}(z \gamma g)=\omega(z) \varphi^{+}(g)
$$

for any $z \in Z^{G}\left(\mathbb{A}_{F}\right), \gamma \in G(F), g \in H^{+}$, and moreover (using $\dot{x}$ to denote $f(x)$ )

$$
\varphi^{+}(\zeta \dot{h})=\varphi(c h)=\omega_{1}(c) \varphi(h)
$$


whenever $\zeta=\dot{c} \gamma$ with $c \in Z_{1}, \gamma \in G(F)$ and $h \in H\left(\mathbb{A}_{F}\right)$. This yields a bijection

$$
L^{2}\left(H(F) \backslash H\left(\mathbb{A}_{F}\right), \omega_{1}\right) \stackrel{\sim}{\longrightarrow} L^{2}\left(G(F) \backslash H^{+}, \omega\right),
$$

that preserves cuspidality. Here cuspidality for representations of $H^{+}$has the obvious definition namely the vanishing of integrals over quotients $U(F) \backslash U\left(\mathbb{A}_{F}\right)$ of non trivial "unipotent subgroups" that are isomorphic images in $G(F) \backslash H^{+}$of quotients of unipotent subgroups in $H\left(\mathbb{A}_{F}\right)$. Hence one obtains a bijection between the cuspidal spectra

$$
L_{\text {cusp }}^{2}\left(H(F) \backslash H\left(\mathbb{A}_{F}\right), \omega_{1}\right) \stackrel{\sim}{\longrightarrow} L_{\text {cusp }}^{2}\left(G(F) \backslash H^{+}, \omega\right) .
$$

It is known that the right regular representation $\rho_{\text {cusp }, \omega_{1}}$ of $H\left(\mathbb{A}_{F}\right)$ in

$$
L_{\text {cusp }}^{2}\left(H(F) \backslash H\left(\mathbb{A}_{F}\right), \omega_{1}\right)
$$

splits into a direct Hilbert sum with finite multiplicities. This implies that the right regular representation of $H^{+}$in $\rho_{\text {cusp }, \omega}^{+}$in $L_{\text {cusp }}^{2}\left(G(F) \backslash H^{+}, \omega\right)$ also splits into a direct Hilbert sum with finite multiplicities.

Now we observe that $L^{2}\left(G(F) \backslash H^{+}, \omega\right)$ is the space of the representation

$$
\rho_{\omega}^{+}=\operatorname{Ind}_{Z^{+}}^{H^{+}} \omega,
$$

while $L^{2}\left(G(F) \backslash G\left(\mathbb{A}_{F}\right), \omega\right)$ is the space of the representation

$$
\rho_{\omega}=\operatorname{Ind}_{Z^{+}}^{G\left(\mathbb{A}_{F}\right)} \omega .
$$

Thus, since induction preserves cuspidality, we see that

$$
\rho_{\text {cusp }, \omega}=\operatorname{Ind}_{H^{+}}^{G\left(\mathbb{A}_{F}\right)} \rho_{\text {cusp }, \omega}^{+} .
$$

\subsection{Main results.}

Theorem 5.2.1. The restriction to $\mathbf{N} \backslash H\left(\mathbb{A}_{F}\right)$ of any cuspidal representation $\pi$ of $G\left(\mathbb{A}_{F}\right)$ contains a cuspidal representation $\sigma$ of $H\left(\mathbb{A}_{F}\right)$.

Proof. Any cuspidal automorphic representation $\pi$ of $G\left(\mathbb{A}_{F}\right)$ with central character $\omega$ occurs in

$$
\rho_{\sigma^{+}}=\operatorname{Ind}_{H^{+}}^{G\left(\mathbb{A}_{F}\right)} \sigma^{+}
$$

for some constituent $\sigma^{+}$of $\rho_{\text {cusp }, \omega}^{+}$. It follows from Lemma 5.1.1 and Proposition 2.3 .3 that $\sigma^{+}$occurs in the restriction of $\pi$ to $H^{+}$. But the isomorphism ( $\star$ ) shows that the restriction of $\sigma^{+}$to $\mathbf{N} \backslash H\left(\mathbb{A}_{F}\right)$ is a direct sum of cuspidal representations.

Theorem 5.2.2. Any cuspidal representation $\sigma$ of $H\left(\mathbb{A}_{F}\right)$ that can be realized in a space of functions on $\Gamma \backslash H\left(\mathbb{A}_{F}\right)$ appears in the restriction of some cuspidal representation $\pi$ of $G\left(\mathbb{A}_{F}\right)$. This is in particular true for any cuspidal representation of $H\left(\mathbb{A}_{F}\right)$ when $f$ is injective.

Proof. Consider $\mathcal{H}$ the subspace of left $\Gamma$-invariant functions in the space of cuspidal square integrable functions modulo the center

$$
L_{\text {cusp }}^{2}\left(H(F) \backslash H\left(\mathbb{A}_{F}\right), \omega_{0}\right)
$$

where $\omega_{0}$ is the character by which $\sigma$ acts when restricted to the center of $H\left(\mathbb{A}_{F}\right)$, The space of the isotypic component, say $W_{\sigma}$, of $\sigma$ in $\mathcal{H}$ can be decomposed according to characters of $\Gamma \backslash Z_{1}$ with $Z_{1}$ acting on the left. Let $\omega_{1}$ be a character that occurs and consider the subspace $W_{\sigma}\left(\omega_{1}\right)$ of $W_{\sigma}$ cut out by this character. Choose $\omega$ extending $\omega_{1}$ to $Z^{+}$. Then $W_{\sigma}\left(\omega_{1}\right)$ can be mapped into a subspace of $\rho_{\text {cusp }, \omega}^{+}$via $(\star)$ and let $\sigma^{+}$be an irreducible constituent of the 
subspace generated by the image of $W_{\sigma}\left(\omega_{1}\right)$ under the action of $H^{+}$. We get a family of cuspidal representations for $G$ by decomposing the induced representation

$$
\rho_{\sigma^{+}}=\operatorname{Ind}_{H^{+}}^{G\left(\mathbb{A}_{F}\right)} \sigma^{+}
$$

According to 2.2.2 the various representations that occur in $\rho_{\sigma^{+}}$are of the form $\pi \otimes \chi$ for some $\pi$ where $\chi$ runs over characters of $G\left(\mathbb{A}_{F}\right) / H^{+}$. Thanks to Lemma 5.1.1 and Proposition 2.3.3 we know that $\sigma^{+}$occurs in the restriction of $\pi$ to $H^{+}$and, in turn, by construction, $\sigma$ occurs in the restriction of $\sigma^{+}$to $\mathbf{N} \backslash H\left(\mathbb{A}_{F}\right)$. The last statement follows from 5.1.2.

\subsection{A reformulation.}

Definition 5.3.1. We denote by $\mathcal{A}_{G}\left(H, \mathbb{A}_{F}\right)$ the set of $G\left(\mathbb{A}_{F}\right)$-conjugacy classes of irreducible unitary representations of $H\left(\mathbb{A}_{F}\right)$ trivial on $\mathbf{N}$.

Definition 5.3.2. Two irreducible unitary representations $\pi$ and $\pi^{\prime}$ of $G\left(\mathbb{A}_{F}\right)$ are said to be $\mathcal{E}_{H}$-equivalent if there exists a character $\mu$ of $G\left(\mathbb{A}_{F}\right) / H\left(\mathbb{A}_{F}\right)$ such that $\pi \otimes \mu \cong \pi^{\prime}$. We denote by $\mathcal{E}_{H}\left(G, \mathbb{A}_{F}\right)$ the corresponding set of equivalence classes.

All elements in the $\mathcal{E}_{H}$-class of some global $\pi$ have equivalent restrictions to $H\left(\mathbb{A}_{F}\right)$ and all components of the restriction belong to the same $G$-packet. Let

$$
R: \mathcal{E}_{H}\left(G, \mathbb{A}_{F}\right) \rightarrow \mathcal{A}_{G}\left(H, \mathbb{A}_{F}\right)
$$

be the map which assigns to an $\mathcal{E}_{H}$-equivalence class represented by $\pi$ the $G\left(\mathbb{A}_{F}\right)$-packet of components of $\left.\pi\right|_{H\left(\mathbb{A}_{F}\right)}$. Observe that $R$ is the restricted product of local restrictions. This makes sense since, for almost all places $v$, the restriction to $H_{v}$ of an unramified representation of $G_{v}$ contains a unique constituent that is unramified.

Proposition 5.3.3. The map

$$
R: \mathcal{E}_{H}\left(G, \mathbb{A}_{F}\right) \rightarrow \mathcal{A}_{G}\left(H, \mathbb{A}_{F}\right)
$$

is a bijection.

Proof. The local analogue 4.1.1implies the injectivity of $R$. The surjectivity follows from the local analogue and the fact that if $G_{v}$ and $H_{v}$ are unramified any unramified representation of $H_{v}$ occurs in the restriction of an unramified representation of $G_{v}$.

We denote by $\mathcal{A}_{G \text {,cusp }}\left(H, \mathbb{A}_{F}\right)$ the subset of $\mathcal{A}_{G}\left(H, \mathbb{A}_{F}\right)$ of $G$-packets that contain some cuspidal automorphic representation of $H\left(\mathbb{A}_{F}\right)$.

We define $\mathcal{E}_{H, \text { cusp }}\left(G, \mathbb{A}_{F}\right)$ to be the subset of $\mathcal{E}_{H}\left(G, \mathbb{A}_{F}\right)$ of $\mathcal{E}_{H}$-equivalence classes that contain some cuspidal automorphic representations of $G\left(\mathbb{A}_{F}\right)$.

Theorem 5.3.4. Assume that $\Gamma=\mathbf{N} . H(F)$ (this is true in particular when $f$ is injective). The map

induces a bijection

$$
R: \mathcal{E}_{H}\left(G, \mathbb{A}_{F}\right) \rightarrow \mathcal{A}_{G}\left(H, \mathbb{A}_{F}\right)
$$

$$
\mathcal{E}_{H, \text { cusp }}\left(G, \mathbb{A}_{F}\right) \stackrel{\sim}{\longrightarrow} \mathcal{A}_{G, \text { cusp }}\left(H, \mathbb{A}_{F}\right) .
$$

Proof. In view of Propositions $4.2 .3,5.3 .3$ and Remark 5.1 .2 this is nothing but a reformulation of Theorems 5.2 .1 and 5.2 .2

Observe that when $\Gamma$ is strictly bigger than $\mathbf{N} \cdot H(F)$ (in particular $f$ is not injective) the map

$$
\mathcal{E}_{H, \text { cusp }}\left(G, \mathbb{A}_{F}\right) \rightarrow \mathcal{A}_{G, \text { cusp }}\left(H, \mathbb{A}_{F}\right)
$$

may not be surjective: an example is given in Remark 5.1.2. The image consists of classes of cuspidal representations that can be realized in a subspace of $\Gamma$-left-invariant functions. 
Remarks 5.3.5. The reader should be aware of the following pitfalls.

1 - If $\sigma$ is a cuspidal representation of $H\left(\mathbb{A}_{F}\right)$ it is not always the case that all conjugates $\sigma^{g}$ for $g \in G\left(\mathbb{A}_{F}\right)$ are automorphic. Examples of this fact do occur in the case $H=S L(n)$ and $G=G L(n)$ for representations that are "endoscopic" (see [16] for the case $n=2$ ).

2 - Consider two cuspidal automorphic representations $\pi$ and $\pi^{\prime}$ that are of the form $\pi^{\prime} \simeq$ $\pi \otimes \mu$; it may happen that $\mu$ cannot be chosen to be automorphic (see [3] where examples are constructed for $H=S L(n)$ and $G=G L(n)$ provided $n \geq 3)$.

5.4. A multiplicity formula. We assume moreover from now on that $f$ is injective. Given an irreducible unitary representation $\pi$ of $G\left(\mathbb{A}_{F}\right)$ the restriction of $\pi$ to $H\left(\mathbb{A}_{F}\right)$ splits into a direct sum with finite multiplicities if $\pi_{v}$ is generic almost everywhere. In fact the restriction to $H_{v}$ of an unramified representation contains a unique constituent that is unramified. The representation $\left.\pi\right|_{H\left(\mathbb{A}_{F}\right)}$ is the direct sum of the restricted products of the constituents of the $\left.\pi_{v}\right|_{H_{v}}$. We know that locally everywhere the multiplicity is finite (cf. 4.1.1). But, whenever $\pi_{v}$ has a Whittaker model, the restriction is multiplicity free. Hence the global decomposition is a direct sum (infinite in general) and with finite multiplicities if $\pi_{v}$ is generic almost everywhere.

We observe that given $\pi$ the set components of $\left.\pi\right|_{H^{+}}$is finite according to Propositions 2.3.1 and 5.1.1 but one should be aware that not all such representations will show up in $\rho_{\text {cusp }, \omega}^{+}$. In fact, for example, if $G=G L(n)$ only one such $\sigma^{+}$, in the restriction to $\mathrm{H}^{+}$ of a given $\pi$, may occur in $\rho_{\text {cusp }, \omega}^{+}$since otherwise this would contradict the multiplicity one theorem for cuspidal representations of $G L(n)$. On the other hand there may be more than one $\sigma^{+}$in the space generated by the isotypic component of some $\sigma$ and they may be inequivalent. This is in fact the case when considering cuspidal representations of $S L(n)$ with multiplicity greater than one (which may exist for $n \geq 3$ ). In such a case the various $\pi$ 's containing $\sigma$ in their restriction to $H\left(\mathbb{A}_{F}\right)$ may differ by non automorphic characters (see [3]). More generally we have the following multiplicity formula.

Theorem 5.4.1. Assume $G$ and $H$ quasi-split. Let $\pi$ be a generic cuspidal representation for $G$ and $\sigma$ a generic cuspidal representation for $H$ that occurs in the restriction of $\pi$ to $H\left(\mathbb{A}_{F}\right)$. Let $Y(\pi)$ be the group of characters $\mu$ of $G\left(\mathbb{A}_{F}\right) / Z^{G}\left(\mathbb{A}_{F}\right) H\left(\mathbb{A}_{F}\right)$ such that $\pi \otimes \mu$ is also a cuspidal representation. Let $X_{l o c}(\pi)$ the subgroup of characters $\mu \in Y(\pi)$ such that $\pi \otimes \mu \simeq \pi$. This is the restricted product over the set of places of $F$ of the $X\left(\pi_{v}\right)$. Let $m(\pi)$ be the multiplicity of $\pi$ in the cuspidal spectrum for $G$. Then, the multiplicity $m(\sigma)$ of $\sigma$ in the cuspidal spectrum of $H$ is given by

$$
m(\sigma)=\sum_{\mu \in M(\pi)} m(\pi \otimes \mu)
$$

where $M(\pi)=Y(\pi) / X_{\text {loc }}(\pi) . X$.

Proof. The uniqueness of Whittaker models tells us that the restriction of $\pi$ to $H\left(\mathbb{A}_{F}\right)$ is multiplicity free. In particular any $\pi$ defines a unique $\sigma^{+}$in $\rho_{\text {cusp }, \omega}^{+}$and conversely this $\sigma^{+}$ is associated to the set of cuspidal representations of the form $\pi \otimes \chi$ with $\chi \in X$ i.e. trivial on $H^{+}$, in particular $\chi$ is automorphic. Now the set of representations $\pi^{\prime}$ in $\rho_{\text {cusp }, \omega}$ whose restriction to $H\left(\mathbb{A}_{F}\right)$ contains $\sigma$, is the set of $\pi^{\prime}=\pi \otimes \mu$ with $\mu \in Y(\pi)$.

5.5. Miscellaneous remarks. Assume again $f$ injective. Let $Z_{0}=Z^{G}\left(\mathbb{A}_{F}\right) \cap H\left(\mathbb{A}_{F}\right)$. The group

$$
Z_{1}=Z^{G}\left(\mathbb{A}_{F}\right) G(F) \cap H\left(\mathbb{A}_{F}\right)
$$

is often equal to $Z_{0} \cdot H(F)$. For example, this latter equality holds in the case $G=G L(n)$ and $H=S L(n)$ whenever the local-global principle for $n^{t h}$-roots of unity holds for $F$ and $n$. 
In fact, if $z \gamma \in Z_{1}$ which means $\operatorname{det}(z \gamma)=1$ then $\operatorname{det}(\gamma)$ is locally everywhere an $n^{t h}$-power, and, if the local-global principle holds, this means that $\operatorname{det}(\gamma)$ is itself an $n^{\text {th }}$-power and $\gamma$ can be rewritten as $\zeta . \eta$ with $\zeta \in Z^{G}(F)$ and $\eta \in H(F)$, hence $z \gamma=z_{0} \eta$ with $z_{0} \in Z_{0}$. This shows that, in this case, the new argument is essentially identical to the argument used in [17. Sect. 3].

Transfer results similar to Theorem 5.2.1 and Theorem 5.2.2 for cuspidal automorphic forms, have been obtained by Chenevier [6] under the condition $Z_{1}=Z_{0} \cdot H(F)$.

As observed in the introduction the map $f: H \rightarrow G$ induces a map $\check{f}$ between dual groups which in turn defines a map

$$
H^{1}(\check{f}): H^{1}\left(W_{F}, \check{G}\right) \rightarrow H^{1}\left(W_{F}, \check{H}\right)
$$

between cohomology sets for Weil groups with values in dual groups. The induced correspondence between packets of representations for $H$ to packets for $G$ provided by Langlands functoriality conjectures should fit with 4.1.3 and 5.2.1. If $f$ is injective Proposition 4.2.3 and Theorem 5.3.4 are compatible with lifting Theorems 7.1 and 8.1 in [14].

\section{Appendix A. Central Morphisms}

\section{By Bertrand LEMAIRE}

Let $k$ be a field. Recall that a morphism of algebraic groups $f: H \rightarrow G$ (over $k$ ) is said to be central if the schematic kernel of $f$ is contained in the schematic center of $H$, which means that for any commutative $k$-algebra $A$, we have the inclusion

$$
\operatorname{Ker}\left(f_{A}: H(A) \rightarrow G(A)\right) \subset Z(H(A)),
$$

where $Z(H(A))$ denotes the center of $H(A) 3$ From [4, 22.4] we know that, given a connected reductive $k$-group $G$, the product morphism $Z^{G} \times G_{d e r} \rightarrow G$ where $Z^{G}$ and $G_{d e r}$ are respectively the (set-theoretic) center and the derived group of $G$, is a central $k$-isogeny.

Let $F$ be a global field. We denote by $\mathbb{A}_{F}$ the adèle ring of $F$. If $v$ is a place of $F$ its completion $F_{v}$ is either $\mathbb{R}$ or $\mathbb{C}$ or a non-Archimedean local field (i.e. a finite extension of $\mathbb{Q}_{p}$, resp. $\left.\mathbb{F}_{p}((t))\right)$.

\section{A.1. Surjective maps of tori.}

Lemma A.1.1. Let $f: T \rightarrow S$ be a surjective morphism of tori.

(1) For any place $v$, the group $S\left(F_{v}\right) / f\left(T\left(F_{v}\right)\right)$ is compact.

(2) The group $S\left(\mathbb{A}_{F}\right) / f\left(T\left(\mathbb{A}_{F}\right)\right) S(F)$ is compact.

Proof. We only give a proof for assertion (2), the proof of (1) being essentially the same but simpler. Let $S_{d}$ be the maximal $F$-split subtorus of $S$, and $X\left(S_{d}\right)$ the group of algebraic characters of $S$ (they are all defined over $F$ ). Let us fix a finite place $v$ of $F$, and a uniformizer element $\varpi_{v}$ of the completion $F_{v}$ of $F$ at $v$. The set

$$
S\left(\varpi_{v}\right)=\operatorname{Hom}\left(X(S), \varpi_{v}^{\mathbb{Z}}\right)
$$

is a free abelian group of finite rank, and a co-compact subgroup of $S_{d}\left(F_{v}\right)$. It also naturally identifies with a subgroup of $S_{d}\left(\mathbb{A}_{F}\right)$. Moreover $S_{d}\left(\varpi_{v}\right) \cap S_{d}(F)=\{1\}$ and the group $S_{d}\left(\mathbb{A}_{F}\right) / S_{d}\left(\varpi_{v}\right) S_{d}(F)$ is compact. Now let $\bar{S}=S / S_{d}$. It is an $F$-anisotropic torus, hence

\footnotetext{
${ }^{3}$ Note that if $k$ is of caracteristic $p>0$, a surjective central $k$-morphism (e.g. a central $k$-isogeny) may be inseparable: for example, the map $t \mapsto t^{2}$ from the multiplicative group $\mathbb{G}_{m}$ into itself, with $p=2$.
} 
the group $\bar{S}\left(\mathbb{A}_{F}\right) / \bar{S}(F)$ is compact [23, 3.5], which implies the group $S\left(\mathbb{A}_{F}\right) / S_{d}\left(\mathbb{A}_{F}\right) S(F)$ is compact. Since

$$
S_{d}\left(\mathbb{A}_{F}\right) \cap\left(S_{d}\left(\varpi_{v}\right) S(F)\right)=S_{d}\left(\varpi_{v}\right) S_{d}(F)
$$

we obtain the group $S\left(\mathbb{A}_{F}\right) / S_{d}\left(\varpi_{v}\right) S(F)$ is compact. On the other hand, $f$ induces a surjective $F$-morphism $f_{d}: T_{d} \rightarrow S_{d}$ which sends $T_{d}\left(\varpi_{v}\right)$ onto a sub-lattice of $S_{d}\left(\varpi_{v}\right)$. Hence the group $S\left(\mathbb{A}_{F}\right) / f\left(T_{d}\left(\varpi_{v}\right)\right) S(F)$ is compact. This implies (2).

\section{A.2. Central surjective morphisms of reductive groups.}

Proposition A.2.1. Let $f: H \rightarrow G$ be a surjective central morphism of connected reductive groups.

(1) For any place $v$, the quotient $G\left(F_{v}\right) / f\left(H\left(F_{v}\right)\right)$ is an abelian compact group.

(2) The quotient $G\left(\mathbb{A}_{F}\right) / f\left(H\left(\mathbb{A}_{F}\right)\right) G(F)$ is an abelian compact group.

Proof. As above, we only give a proof of assertion (2). From [5, 2.2, 2.6] (see [5, 2.3]), there exists an $F$-morphism $\kappa: G \times G \rightarrow H$ such that for all $x, y \in H$, we have

$$
\kappa(f(x), f(y))=x y x^{-1} y^{-1} .
$$

So the commutator map $G \times G \rightarrow G,(x, y) \mapsto[x, y]=x y x^{-1} y^{-1}$ coincides with $f \circ \kappa$, and we have

$$
\left[G\left(\mathbb{A}_{F}\right), G\left(\mathbb{A}_{F}\right)\right]=f \circ \kappa\left(G\left(\mathbb{A}_{F}\right) \times G\left(\mathbb{A}_{F}\right)\right) \subset f\left(H\left(\mathbb{A}_{F}\right)\right) .
$$

Hence $f\left(H\left(\mathbb{A}_{F}\right)\right)$ is an invariant subgroup of $G\left(\mathbb{A}_{F}\right)$, and the group $G\left(\mathbb{A}_{F}\right) / f\left(H\left(\mathbb{A}_{F}\right)\right)$ is abelian. A fortiori the quotient $G\left(\mathbb{A}_{F}\right) / f\left(H\left(\mathbb{A}_{F}\right)\right) G(F)$ is an abelian group. It remains to prove the compacity. Let $S$ be a maximal $F$-split torus in $G$, and $M=Z^{G}(S)$ the centralizer of $S$ in $G$. Let $P$ be a (minimal) parabolic $F$-subgroup of $G$ with Levi component $M$, and $U=U_{P}$ the unipotent radical of $P$. From [4, 22.6], the inverse image $S^{\prime}$ of $S$ in $H$ is a maximal $F$-split torus in $H$, and the inverse image $P^{\prime}$ of $P$ in $H$ is a minimal parabolic $F$-subgroup of $H$. Put $M^{\prime}=Z^{H}\left(S^{\prime}\right)$ and $U^{\prime}=U_{P^{\prime}}$. From loc. cit., $f$ induces a surjective $F$-morphism $M^{\prime} \rightarrow M$ and an $F$-isomorphism $U^{\prime} \rightarrow U$. Moreover, $M^{\prime} \rightarrow M$ is central. On the other hand, we have the Iwasawa decomposition $G\left(\mathbb{A}_{F}\right)=\boldsymbol{K} P\left(\mathbb{A}_{F}\right)$ where $\boldsymbol{K}=\prod_{v} \boldsymbol{K}_{v}$ is an $M$-admissible maximal compact subgroup of $G\left(\mathbb{A}_{F}\right)$. Hence the product map $\boldsymbol{K} \times P\left(\mathbb{A}_{F}\right) \rightarrow G\left(\mathbb{A}_{F}\right)$ gives a surjective map

$$
\boldsymbol{K} \times\left(P\left(\mathbb{A}_{F}\right) / f\left(P^{\prime}\left(\mathbb{A}_{F}\right)\right) P(F) \rightarrow G\left(\mathbb{A}_{F}\right) / f\left(H\left(\mathbb{A}_{F}\right)\right) G(F) .\right.
$$

Since $f\left(U^{\prime}\left(\mathbb{A}_{F}\right)\right)=U\left(\mathbb{A}_{F}\right)$, we have

$$
P\left(\mathbb{A}_{F}\right) / f\left(P^{\prime}\left(\mathbb{A}_{F}\right)\right) P(F)=M\left(\mathbb{A}_{F}\right) / f\left(M^{\prime}\left(\mathbb{A}_{F}\right)\right) M(F) .
$$

So we just need to prove the compacity of the quotient (that we already know to be an abelian group) $M\left(\mathbb{A}_{F}\right) / f\left(M^{\prime}\left(\mathbb{A}_{F}\right)\right) M(F)$. Since the quotient $\bar{M}=M / S$ is a connected reductive $F$-anisotropic group, the set $\bar{M}\left(\mathbb{A}_{F}\right) / \bar{M}(F)$ is compact [23, 3.5], which implies the set $M\left(\mathbb{A}_{F}\right) / S\left(\mathbb{A}_{F}\right) M(F)$ is compact. A fortiori the quotient

$$
M\left(\mathbb{A}_{F}\right) / f\left(M^{\prime}\left(\mathbb{A}_{F}\right)\right) S\left(\mathbb{A}_{F}\right) M(F)
$$

is compact. Since

$$
f\left(M^{\prime}\left(\mathbb{A}_{F}\right)\right) \cap\left(S\left(\mathbb{A}_{F}\right) M(F)\right)=f\left(S^{\prime}\left(\mathbb{A}_{F}\right)\right) S(F),
$$

we are reduced to prove the compacity of the group $S\left(\mathbb{A}_{F}\right) / f\left(S^{\prime}\left(\mathbb{A}_{F}\right)\right) S(F)$. It is given by the Lemma A.1.1.

Corollary A.2.2. Let $f: H \rightarrow G$ be an F-morphism of connected reductive groups such that the induced morphism $f_{d e r}: H_{d e r} \rightarrow G_{d e r}$ is a central isogeny. 
(1) For any place $v$, the quotient $G\left(F_{v}\right) / Z^{G}\left(F_{v}\right) f\left(H\left(F_{v}\right)\right)$ is an abelian compact group.

(2) The quotient $G\left(\mathbb{A}_{F}\right) / Z^{G}\left(\mathbb{A}_{F}\right) f\left(H\left(\mathbb{A}_{F}\right)\right) G(F)$ is an abelian compact group.

Proof. The morphism

$$
\text { id } \times f_{\text {der }}: Z^{G} \times H_{\text {der }} \rightarrow Z^{G} \times G_{\text {der }}
$$

and the product morphism $Z^{G} \times G_{d e r} \rightarrow G$ are central $F$-isogenies. The composition of these two morphisms $Z^{G} \times H_{\text {der }} \rightarrow G$ is also a central $F$-isogeny. This implies the corollary.

Remarks A.2.3. In the corollary, we may replace $Z^{G}$ by its connected component $Z$, which is the maximal central F-torus in $G$; the product morphism $Z \times G_{d e r} \rightarrow G$ is still a central F-isogeny.

\section{REFERENCES}

[1] Artin E., Tate J.T., Class field theory. W. A. Benjamin, Inc., New York, Amsterdam, 1968; [Advanced Book Classics, Addison-Wesley Publishing Company, Inc., Redwood City, CA, 1990].

[2] Bernstein I. N., All reductive p-adic groups are of type I. Funkcional. Anal. i Prilozen. 8 (1974), no. 2, 3-6.

[3] Blasius D., On multiplicities for SL(n). Israël Journal of Math. 88 (1994), 237-251.

[4] Borel A., Linear Algebraic Groups, Second Enlarged Edition, Graduate Texts in Math., vol. 126, Springer-Verlag, New York, 1991.

[5] Borel A., Tits J., Compléments à l'article "Groupes Réductifs", Publ. Math. I.H.É.S 41 (1972), $253-276$

[6] Chenevier G., On restrictions and extensions of cusp forms, Private communication

[7] Conrad B., Finiteness theorems for algebraic groups over function fields, Compos. Math. 148 (2012), no. 2, 555-639.

[8] Curtis, C. W. Reiner I., Representation theory of finite groups and associative algebras, A WileyInterscience Publication. John Wiley Sons, Inc., New York, 1988

[9] Gelbart S. S., Knapp A. W., L-indistinguishability and $R$ groups for the special linear group. Adv. in Math. 43 (1982) no. 2, 101-121.

[10] Harder G., Über die Galoiskohomologie halbeinfacher algebraischer Gruppen. III. J. Reine Angew. Math. 274/275 (1975), 125-138.

[11] Henniart G., Représentations des groupes réductifs p-adiques et de leurs sous-groupes distingués cocompacts. Journal of Algebra. 236 (2001), 236-245,

[12] Hiraga K., Saito H., On L-packets for inner forms of $S L_{n}$. Mem. Amer. Math. Soc. 215 (2012), no. 1013,

[13] Котtwitz R., Shelstad D., Foundations of twisted endoscopy, Asterisque 255 (1999)

[14] Labesse J.-P., Cohomologie, L-groupes et fonctorialité, Compositio Math.. 55 (1985), 163-184.

[15] Labesse J.-P., Cohomologie, stabilisation et changement de base, Asterisque 257 (1999)

[16] Labesse J.-P., Langlands R.P., L-indistinguishability for SL(2), Can. J. Math. 31 (1979), 726-785.

[17] Labesse J.-P., Schwermer J., On liftings and cusp cohomology of arithmetic groups, Invent. Math. 83 (1986), 383-401.

[18] Mackey G.W., Induced Representations of Locally Compact Groups II. The Frobenius Reciprocity Theorem, Ann. of Maths. (2), 58 (1953), 193-221.

[19] Milne J.S., Points on a Shimura Variety, Appendix B in "The Zeta Function of Picard Modular Surfaces", Les publications CRM, Montréal (1992), 229-244.

[20] Moore C.C., On The Frobenius Reciprocity Theorem for Locally Compact Groups. Pacific J. of Mathematics, 12 No. 1 (1962), 359-365.

[21] Meghin C., Waldspurger J.-L., Décomposition spectrale et séries d'Eisenstein, Progress in Math. 113 Birkhäuser(1994).

[22] Silberger A., Isogeny restrictions of irreducible admissible representations are finite direct sums of irreducible admissible representations, Proc. Amer. Math. Soc. 73 (1979) No. 2, 263-264.

[23] Springer T. A. Reduction theory over global fields, Proc. Indian Acad. Sci. (Math. Sci.) 104 (1994), 207-216.

[24] TAdic̀ M., Notes on representations of non archimedean $S L(n)$, Pacific J. of Math. 152 No. 2 (1992), 375-396. 
Institut de Mathématique de Marseille, UMR 7373, Aix-Marseille Université, France

E-mail address: Jean-Pierre.Labesse@univ-amu.fr

Faculty of Mathematics, University Vienna, Oskar-Morgenstern-Platz 1, A-1090 Vienna, Austria resp. Max-Planck-Institute for Mathematics, Vivatsgasse 7, D-53111 Bonn, Germany.

E-mail address: Joachim.Schwermer@univie.ac.at 